\title{
UNA COEXISTENCIA RÍTMICA PARA LAS DURACIONES (ENTRE Bergson y DeleuZe) ${ }^{1}$
}

\author{
Cristóbal Durán Rojas ${ }^{2}$ \\ Felipe Kong Aránguiz ${ }^{3}$
}

\begin{abstract}
Resumen: La intención del presente artículo es reexaminar el concepto bergsoniano de Duración entendido como coexistencia de niveles de multiplicidad heterogéneos. Si bien dicho concepto ha sido pensado a partir de una comprensión que podría ser caracterizada como continuista, el mismo Bergson evitará confundir dicha continuidad con la idea de homogeneidad. Intentaremos mostrar que la determinación del concepto de Duración se alcanza al definirla como tensión o entrelazamiento entre sucesión y simultaneidad, y por consiguiente, como una multiplicidad que hace coexistir diferentes flujos de duración. Para ello, Bergson propone la existencia de un Tiempo impersonal y único como base para la relación entre duraciones. Nuestra hipótesis es que esta misma situación puede entenderse mejor si se articula el concepto de ritmo en la obra de Deleuze, concepto ligado íntimamente a las nociones de duración y coexistencia. Mediante los movimientos de contracción, dilatación, reunión y división, el ritmo opera junto a las duraciones disponiéndose como un campo de relación, pudiendo ocupar el lugar que había sido asignado al Tiempo único.
\end{abstract}

Palabras Claves: Duración. Bergson. Deleuze. Coexistencia. Ritmo. Simultaneidad.

\section{LA DURACIÓN Y EL PROBLEMA DE LA CONTINUIDAD}

El concepto de duración tiene una aparición temprana en el corpus de pensamiento bergsoniano. Dicha aparición obedece al intento de elaborar una distinción específica entre el tiempo y el espacio, en el entendido de que la tradición de pensamiento pareciera, según Bergson, haber hecho caso omiso

\footnotetext{
${ }^{1}$ Este trabajo es resultado del Proyecto FONDECYT de Iniciación $\mathrm{N}^{\circ} 11150732$ "El dinamismo singular de la relación: elementos para una reconstrucción de la ontología relacional a partir de una teoría de las multiplicidades (Simondon, Deleuze)”. Investigador responsable: Dr. Cristóbal Durán Rojas.

${ }^{2}$ Doctor en Filosofía con mención en Estética y Teoría del arte, Universidad de Chile. Actualmente es profesor asociado en la Universidad Andrés Bello, Facultad de Humanidades y Ciencias Sociales. Fernández Concha 700, Las Condes, Santiago, Chile. Mail: cristobal.duran@unab.cl. Es coordinador del Núcleo de Teoría de las Multiplicidades. Recientemente ha publicado Polifonías. Jacques Derrida: la voz, la sorpresa (Das Kapital, 2017).

http://dx.doi.org/10.1590/S0101-317320170002000010

${ }^{3}$ Doctor (c) en Filosofía con mención en Estética y Teoría del arte, Universidad de Chile. Mail: felipekaranguiz@gmail.com. Actualmente prepara la publicación de su libro La corte confusa. Walter Benjamin y el Tarot, que será publicado durante 2017. Es miembro del Núcleo de Teoría de las Multiplicidades.
} 
respecto a dicha distinción. En esa medida, todo el plan presentado en el Essai sur les données immédiates de la conscience, publicado en 1889, está elaborado con el objetivo de hacer justicia a una serie de distinciones conceptuales que parecieran arrastrar consigo una mezcla de elementos que realmente se diferencian en naturaleza. Tanto el tiempo como el espacio serían en estricto rigor mixtos mal analizados, que deberían ser distinguidos con precisión, pues de lo contrario, se corre el riesgo de confundir la heterogeneidad de elementos que componen dichos conceptos, reduciéndolos bajo la ilusión que los considera como totalidades homogéneas. De este modo, al interrogar el tiempo o el espacio, no sería extraño confundir en su análisis diferencial elementos como la sucesión y la simultaneidad, la duración y la extensión, o la cualidad y la cantidad (BERGSON, 1991, p. 180).

Ciertamente no se trata sólo de denunciar una confusión, sino de poner en entredicho el hecho de que esa confusión se eleve a estatuto de concepto pretendidamente nítido y determinado. Es por esa razón que, en la terminología bergsoniana, la duración no se identifica plenamente con el

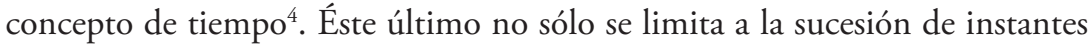
e intervalos que pueden ser medidos y considerados a partir de dicha medida como parte de una continuidad; dicha sucesión también implica la vivencia de los intervalos, es decir, la intuición de la duración. Se trata, como recuerda una definición clásica del Essai, de considerar a la duración totalmente pura como “[...] la forma que adquiere la sucesión de nuestros estados de conciencia cuando nuestro yo se deja vivir, cuando se abstiene de establecer una separación entre el estado presente y los estados anteriores." (BERGSON, 1991, p. 75).

Una definición de duración como la recién citada se mantiene explícitamente ligada a la sucesión, lo que concuerda perfectamente con el énfasis puesto en la "continuidad ininterrumpida" que aproxima la descripción de la duración al paradigma de la escucha de una melodía (BERGSON, 1990a, p. 164). El uso de la melodía sirve metafóricamente para dar cuenta de una posibilidad de escapar a toda representación en un espacio homogéneo, dado que, pese las diferencias entre las notas, "[...] las notas de una melodía

\footnotetext{
${ }^{4}$ Es cierto que el mismo Bergson advierte que al determinar el concepto de duración, por contraste con el tiempo, la mayor dificultad reside en que sus escritos no consideran un único concepto de tiempo, y con ello no bastaría sencillamente con denunciar al tiempo como un mixto confuso entre duración y espacio extensivo. Al decir de Bergson: "Parto de la 'duración' e intento esclarecer este problema, sea por contraste, o bien por similitud con él. Lamentablemente, mis libros no siempre son coherentes entre sí: el 'tiempo' de la Évolution créatrice no 'calza' con el de Données immédiates." (apud BÉGUIN; THÉVENAZ, 1943, p. 360).
} 
son percibidas como un todo, sin referencia al espacio." (CORBIER, 2012, p. 297). Sin embargo, esta vía de interpretación del concepto de duración que podríamos denominar continuista- responde a un modo de poner énfasis sobre una consideración de una multiplicidad no espacial, que es la que se construye a partir del examen de la naturaleza de los hechos psíquicos. La propuesta de una duración interior, elaborada en el Essai, tiene que poner en suspenso el carácter discontinuo de la simultaneidad con el objeto de dar cuenta de la duración como "[...] la vida continua de una memoria que prolonga el pasado en el presente.” (BERGSON, 1990a, p. 200-201).

Pero el problema de la continuidad de la duración no impide que aquella sea caracterizada a partir de una heterogeneidad en las dimensiones que la componen. Una multiplicidad diferente de la que pueda ser conceptualizada en términos de su distinción espacial, se hace necesaria una vez que se trata de dar cabida a la diferencia de partes que constituyen y organizan lo que es percibido como un todo. De este modo, sería la heterogeneidad (por ejemplo, de las notas de una melodía) lo que permite la sucesión de las notas, y finalmente, lo que otorgaría cierta disimetría a la continuidad duracional 5 .

\section{MulTiPLICIDAD Y RITMOS DE DURACIÓN}

Frente a una comprensión continuista, que corre el riesgo de reducir la continuidad a la homogeneidad, y que hace caso omiso de la heterogeneidad continua con la que se intenta caracterizar la duración, la labor del segundo capítulo del Essai consistirá en argumentar a favor de una distinción entre dos tipos de multiplicidades, que sería finalmente requerida para determinar la mixtura que se lleva a cabo entre tiempo y espacio. Dado que los hechos psíquicos coexisten en nuestra conciencia, pero no a la manera de elementos que difieran en magnitud (BERGSON, 1991, p. 7), es preciso mostrar que la variación y el cambio de los estados afectivos que determinan nuestra actividad psíquica parecen ceñirse a la idea de una intensidad que exige pensar la pura duración de los estados de conciencia, considerada a partir de una

\footnotetext{
${ }^{5}$ No es preciso ir tan lejos como Bachelard, quien intentó desarrollar un "bergsonismo discontinuo", con el cual proponía otorgar mayor fluidez y articulación al concepto de duración (BACHELARD, 2013, p. 7-8). En La dialectique de la durée, discutirá precisamente la tesis de la continuidad en la filosofía de la duración con el objeto de "[...] establecer metafísicamente la existencia de lagunas en la duración" (BACHELARD, 2013, p. VII). La interrupción del continuo no es exterior a la duración, y según su argumento dice relación con el hecho de considerar que "la duración es metafísicamente compleja y los centros decisivos del tiempo son sus discontinuidades." (BACHELARD, 2013, p. 38).
} 
forma obtenida "[...] cuando se hace abstracción del espacio en que aquella se despliega.” (BERGSON, 1991, p. 55).

Bergson expondrá, así, que es preciso dar cuenta de una multiplicidad que nos permita hacer sensible la duración de estados que son sucesivos y que, aunque diferentes entre sí, no son susceptibles de ser pensados mediante una yuxtaposición. Se denuncia la yuxtaposición, ya que ella pareciera originarse en una manera de superponer una tesis espacial al tiempo, que es imprecisa y poco rigurosa, y que no permite advertir la duración de elementos no discretos y que supondrían una continuidad diferenciada (BERGSON, 1991, p. 57). La variación de estados psíquicos no podría entonces ser pensada como una operación de cuenta de objetos exteriores entre sí, ya que ello implicaría una representación simultánea de dichos objetos que son así abstractamente mantenidos -y dados de golpe- en el espacio. Y de este modo, sólo nos figuraríamos la separación entre instantes, pero perderíamos lo que constituye propiamente su duración.

La multiplicidad propia de la duración interior no es producto de una adición de estados, que realmente serían percibidos simultáneamente y dados como un todo compuesto de partes. Las sensaciones, ideas y sentimientos se compenetran entre sí, de modo tal que unos se superponen con otros, sin necesidad de ser considerados como "unidades homogéneas" (BERGSON, 1991, p. 67) que se dejen representar simbólicamente ocupando distintos lugares extensivos. Si el tiempo es concebido como un medio homogéneo que contiene estados de conciencia, no seríamos capaces de experimentar el cambio implicado en la duración, dado que consideramos dicha unión entre tiempo y estados de una sola vez. Ello implicaría pensar, como advierte Bergson, que los hechos de conciencia sean captados como intervalos exteriores unos de otros, "[...] pero [en realidad] los hechos de conciencia, incluso sucesivos, se penetran y en el más simple de ellos puede reflejarse el alma entera." (BERGSON, 1991, p. 73).

Ambas formas de entender la multiplicidad están ligadas entre sí y se presentan mezcladas, de tal modo que una duración homogénea, que no es más que el "[...] símbolo extensivo de la duración verdadera" (BERGSON, 1991, p. 94), presupone una duración cuyos momentos heterogéneos se penetran entre sí y que se define mediante una sucesión que implica fusión y organización. Dicha penetración mutua cualitativa, dice Bergson, hace posible la adición, modificando "[...] la naturaleza, el aspecto y como el ritmo del conjunto.” (BERGSON, 1991, p. 92). Es importante advertir que para 
producir una multiplicidad indistinta o cualitativa - imagen de una duración pura- es preciso que la cualidad dada por la organización rítmica del conjunto sea sustraída de la imagen de la yuxtaposición. Los elementos que definen dicha organización rítmica se mezclan de tal manera que no se podría decir si son uno o muchos, y al no constituir una multiplicidad numérica, su duración no se basaría en la extensión distinta de unos sobre otros (BERGSON, 1991, p. 102).

Pese a que la distinción entre ambos tipos de multiplicidad permite distintos modos de pensar la sucesión y la simultaneidad (BERGSON, 1991, p. 81-82), al considerar al espacio como lo que no dura, las simultaneidades interiores se oponen a la discontinuidad espacial. Sólo al publicar Matière et mémoire, escrito en 1896, se puede plantear un intento de entrelazar ambas, ya no subordinando parcialmente la multiplicidad cualitativa a la multiplicidad cuantitativa de los objetos espaciales. En dicho texto, lo cuantitativo ya no es exterior a lo cualitativo, y la diferencia entre ambas responde más bien a diferencias de tensión de la duración misma. Entre las cualidades sensibles tal como aparecen en nuestra representación y ellas consideradas como cambios calculables, no habría más que una "diferencia de ritmo de duración" o una “diferencia de tensión interior" (BERGSON, 1990b, p. 278-279). Tensión y extensión admiten grados múltiples, lo que permitirá no limitar la extensión a una determinación esencial de los elementos discretos y discontinuos que se disponen en el espacio. Considerada como la forma más tensa del movimiento, la duración no sería una mera continuidad opuesta a la discontinuidad, sino que abriría la pregunta sobre el modo de captar la heterogeneidad de la multiplicidad real como una implicación entre la duración continua y la discontinuidad efectiva de duraciones que difieren entre sí.

La duración, en cuanto multiplicidad, no puede ser discreta, dado que es una composición de ritmos de duración y no un conjunto de instantes o puntos temporales. Pero, por otro lado, tampoco se puede limitar a adoptar una forma continua, ya que ello impediría elaborar distinciones entre duraciones, que son las que afirman la multiplicidad. Esto porque "[...] no hay un ritmo único de la duración; podemos imaginar varios ritmos diferentes que, más lentos o más rápidos, medirían el grado de tensión o de relajación de las conciencias, y, por consiguiente, fijarían sus lugares respectivos en la serie de los seres." (BERGSON, 1990b, p. 232). Esto supondría pensar algún grado de coexistencia posible entre las conciencias que duran, un grado que ya no correspondería evidentemente a la relación que se puede establecer en un 
conjunto de elementos distintos, ya que las duraciones -o las diferencias de ritmo de la duración - son en cada caso absolutas, y no elementos individuados que pudieran ser luego yuxtapuestos siguiendo una regla homogénea de unificación.

\section{LA SIMULTANEIDAD EN LA DURACIÓN Y LA HIPÓTESIS DE LA COEXISTENCIA}

La necesidad de replantearse la coexistencia entre diferentes ritmos de duración desemboca en una revalorización de la noción de simultaneidad. Si bien ésta última era considerada como una relación espacial que distinguía estados y que era concebida como lo único fuera de nuestra conciencia (BERGSON, 1991, p. 170), dicha comprensión se rearticula y se transforma en un problema central en Durée et simultanéité, de 1922. En dicho texto, la simultaneidad consiste más bien en "[...] la posibilidad para dos o varios acontecimientos de entrar en una percepción única e instantánea” (BERGSON, 1968, p. 43), lo que parece asociar la simultaneidad a la unidad dada por la percepción. Más todavía, se afirmará la necesidad de distinguir entre dos especies de simultaneidad. "La primera es interior a los acontecimientos; ella forma parte de su materialidad y viene de ellos. La otra especie simplemente está puesta sobre ellos por un observador externo al sistema." (BERGSON, 1968, p. 93).

¿A qué se refiere Bergson con una simultaneidad interior a los acontecimientos? No es una impuesta por un observador externo al sistema, sino que es la capacidad de distintos acontecimientos de entrar en una percepción única e instantánea. Lo que quiere decir que no se trata de una simultaneidad entre instantes, sino entre continuos que coexisten en su duración. Se trata de una idea que Bergson califica como más natural, y que es la de cierta simultaneidad de flujos:

Los teóricos de la Relatividad no hablan más que de la simultaneidad de dos instantes. Antes de esta, hay sin embargo otra, cuya idea es más natural: la simultaneidad de dos flujos. Decimos que es parte de la esencia misma de nuestra atención poder repartirse sin dividirse. Cuando estamos sentados a orillas de un río, el flujo del agua, el deslizamiento de un barco o el vuelo de un pájaro y el murmullo ininterrumpido de nuestra vida profunda son para nosotros tres cosas diferentes o una sola, a voluntad. [...] Esa es nuestra primera idea de simultaneidad. Denominamos simultáneos a dos flujos exteriores que ocupan la misma duración porque ellos se sostienen en la duración de un tercero, el nuestro: esta duración es sólo la nuestra 
cuando nuestra conciencia nos mira sólo a nosotros, sino que ella se convierte igualmente en la nuestra cuando nuestra atención abraza los tres flujos en un único acto indivisible. (BERGSON, 1968, p. 50-51).

Esta simultaneidad de flujos no hace más que dar cuenta de un apuntalamiento de duraciones, apoyándose en una atención que es capaz de "repartirse sin dividirse". De este modo, la indivisible movilidad de la duración (p. 47) requiere la posibilidad de distinguir flujos que la configuran. Por ello, la simultaneidad que ahora se considera para dar cuenta de la duración, permite reconocer a esta última no como lo "[...] simplemente indivisible, sino como lo que tiene un estilo muy particular de división; ella no es simplemente sucesión, sino coexistencia muy particular, simultaneidad de flujos." (DELEUZE, 1966, p. 81 , el subrayado es nuestro). Responde, más bien, a la necesidad de dar cuenta de un lugar de entrecruzamiento entre duración y extensión. De ahí que se pueda afirmar que el problema planteado por Bergson no es tanto el del continuo como el de la conexión: "Lo que Bergson quiere que reconozcamos no es la presencia de una duración universal que envolvería a todas las demás o que las escandiría como un bajo continuo, sino la conexión que permite decir que las duraciones, por muy diversamente ritmadas que uno se las figura, coexisten en un mismo mundo." (DURING, 2008, p. 149).

En este sentido, Bergson elabora un concepto de tiempo capaz de integrar la duración del universo y la medida de las duraciones de las conciencias individuales, aspirando a no subsumir unas bajo la otra. Si la duración "[...] es esencialmente una continuación de lo que ya no es en lo que es" (BERGSON, 1968, p. 46), es preciso buscar un modo de hacer coexistir la indistinción entre una duración que separa dos instantes, y la memoria que liga dichos instantes. A un tiempo impersonal e universal, que se prolonga sin fin y que es "todo de una pieza" (BERGSON, 1968, p. 48), responde la evidencia de duraciones diferentes, definidas por ritmos diversos. Ese tiempo único es un tiempo real, en la medida en que nos permite la distinción de momentos y niveles en él, y nos da la posibilidad a cada instante de confrontar la división de lo desplegado al hecho mismo del despliegue. Y esto supone afirmar que, pese a su posibilidad de ser dividido, el tiempo real no se compone de instantes. Pero es efectivamente medido como un tiempo "[...] susceptible de ser retomado en su flujo por una conciencia que vive y dura." (DURING, 2009, p. 238). Corresponde, más bien, a la percepción instantánea de un conjunto, percepción única que es sin embargo múltiple y divisible en cada caso. 
Es preciso afirmar que los acontecimientos no dependen del punto de vista desde donde se los considere para ser comprendidos como simultáneos; se trata, más bien, de plantear en el problema de la simultaneidad la posibilidad de un tiempo impersonal no homogéneo. Es en ese sentido que se puede afirmar que el tema de la simultaneidad abre la filosofía de Bergson como una filosofía de la coexistencia: un tiempo uno y múltiple, en tanto la duración es sucesión si, y sólo si, puede dar cuenta de una coexistencia virtual que, como Deleuze lo ha advertido, es rigurosamente una "[...] coexistencia consigo de todos los niveles, de todas las tensiones, de todos los grados de contracción y de distensión" (BERGSON, 1966, p. 56), y que confirman que una teoría de la multiplicidad revela a la duración como coexistencia de todos los grados "[...] en un solo y mismo tiempo." (BERGSON, 1966, p. 86).

Ahora bien, si la simultaneidad es la que permite calificar al tiempo como un todo (DELEUZE, 1983, p. 69), es preciso reconocer un tiempo único escandido diferencialmente, por así decir, ritmado diferencialmente. Así se podría entender, como señala Deleuze en Le Bergsonisme, que no se puede renunciar ni a la idea de una infinidad de flujos actuales, ni a las diferencias de distensión y contracción en la virtualidad que los envuelve. Bergson "[...] estima que estas dos certezas no se excluyen, y que por el contrario ellas implican un tiempo único. En resumen: no sólo las multiplicidades virtuales implican un único tiempo, sino que la duración como multiplicidad virtual es ese único y mismo Tiempo.” (DELEUZE, 1966, p. 84). En la medida en que la duración no está dada, que ella no es un todo homogéneo, pero que, al llevar a cabo intentos de segmentación de ella, no hace más que exponer otra duración, se podría decir que hay más bien una relación inmanente (o una relación de diferencia inclusiva) entre diferencias de duración o "ritmos de duración" (que son a su vez, duraciones que difieren). Siempre hay duración y sus otros, sus capturas, por ejemplo, pero el problema es siempre cómo pensar esas diferencias, esas relaciones diferenciales.

\section{Gilles DeleuZE: ¿̨UNO O VARIOS RITMOS?}

Hemos visto que la noción de ritmo ha acompañado a la duración en momentos clave del texto bergsoniano, a pesar del casi nulo desarrollo teórico de este concepto en su obra. Sin embargo, para Deleuze el ritmo sí cobra relevancia, y de un modo que muchas veces se entrecruza con los conceptos de duración, diferencia y coexistencia, por mencionar algunos de clara raigambre bergsoniana. Por ello, nos interesa articular brevemente esta 
noción en la obra deleuziana, en vistas a determinar sus posibles alcances para una clarificación del concepto de duración que conceda un lugar central a la noción de coexistencia de diferencias.

La noción de ritmo en Deleuze aparece dispersa en distintos textos, tomando formas muy disímiles que a veces parecen contradecirse. A pesar de tener una especial relevancia en el capítulo "De la ritournelle" en Mille Plateaux o en Logique de la sensation, no ha sido considerado como un concepto importante en la obra deleuziana: podría decirse que es un concepto menor, tomando en cuenta su poca presencia en los vocabularios que existen sobre ella ${ }^{6}$. Únicamente nos centraremos en algunos pasajes que tienen una relevancia especial para la relación entre duración y coexistencia, que es lo que nos convoca en este trabajo.

Si queremos encontrar una primera tematización directa del ritmo en la obra del filósofo francés hay que acudir a Différence et répétition. Allí Deleuze expone en un pasaje sobre la existencia de dos tipos de repetición: la repeticiónmedida y la repetición-ritmo. En la primera se trabaja con un tiempo dividido regularmente, con puntos idénticos y equidistantes; en la segunda, en tanto, hay intensidades que van "[...] creando desigualdades, inconmensurabilidades, en duraciones o espacios métricamente iguales." (DELEUZE, 1993, p. 33). Pero no se trata de que los ritmos vengan a actuar sobre un espacio-tiempo medido, sino más bien al revés. Los ciclos de repetición de lo Mismo no son más que envolturas de ritmos complejos que funcionan como movimientos más profundos:

La reiteración de puntos de desigualdad, de puntos de flexión, de acontecimientos rítmicos, es más profunda que la reproducción de elementos ordinarios homogéneos; de modo que, en todos los casos, debemos distinguir la repetición-medida y la repetición-ritmo, ya que la primera es sólo la apariencia o el efecto abstracto de la segunda. (DELEUZE, 1993, p. 33).

Pero ¿cómo podemos entender esta profundidad? ¿En qué se sustenta la existencia de estos ritmos, más allá de la medida? Más adelante, Deleuze

\footnotetext{
${ }^{6}$ Véase ZOURABICHVILI (2003); SASSO ; VILLANI (2003); PARR (2005); YOUNG (2013). Sólo en este último hay una entrada de una página y media para "ritmo", que se basa sobre todo en el texto sobre el ritornelo. Pero cuando decimos que es un concepto "menor", estamos aludiendo también al sentido que esta palabra tiene en Deleuze y Guattari: "[...] el modo mayor y el modo menor son dos tratamientos de la lengua: uno consiste en extraer constantes, el otro en ponerlas en variación continua." (DELEUZE; GUATTARI, 1980, p. 130).
} 
volverá a hablar de ritmos para expresar el paso del tiempo virtual al tiempo actual mediante un proceso de diferenciación. Si bien las estructuras poseen todas "un tiempo puramente lógico, ideal o dialéctico", "[...] ese mismo tiempo virtual determina un tiempo de diferenciación [différenciation] o más bien ritmos, tiempos diversos de actualización que corresponden a las relaciones y a las singularidades de la estructura." (DELEUZE, 1993, p. 272). El ritmo no es sólo diferenciador en relación con la medida, que en todo caso es efecto de él; el ritmo es en sí mismo diferenciación, motor de actualización múltiple a partir de lo virtual. Por ello Deleuze hablará en otras partes del libro de "ritmo diferencial".?

Así, ya tempranamente el ritmo se dispone entre dos niveles: antes de la medida, que funciona como una determinación abstracta de éste, y después de lo virtual que lo engendra como diferenciación. Este nivel pre-rítmico será llamado en Mille Plateaux el Caos: "Del caos nacen los Medios y los Ritmos" (DELEUZE; GUATTARI, 1980, p. 384). Si bien "[c]ada medio es vibratorio, es decir, un bloque de espacio-tiempo constituido por la repetición periódica de la componente" (DELEUZE; GUATTARI, 1980, p. 384), esta repetición no implica en sí misma una ritmicidad, como ya hemos visto. El ritmo es "[...] la respuesta de los medios al Caos" (DELEUZE; GUATTARI, 1980, p. 385), el intercambio de sus códigos que constituye de por sí un ser distinto al de los medios. El ritmo es un ser-entre: "Lo que tienen de común el caos y el ritmo es el entre-dos, entre dos medios, ritmo-caos o caosmos [...] En ese entre-dos el caos deviene ritmo, no necesariamente, pero tiene una posibilidad de devenirlo. El caos no es lo contrario del ritmo, más bien es el medio de todos los medios." (DELEUZE; GUATTARI, 1980, p. 385). Así, conjuntamente con comprenderse como diferenciación, el ritmo se entiende aquí como relación. Pero no es una relación que dependa de sus términos - los medios -, a pesar de que en este pasaje se señale que el ritmo surge de ellos. Cada ritmo singular, esto es, cada relación de transcodificación entre medios, se origina evidentemente en ese preciso encuentro. Pero lo rítmico en general, es decir, el ritmo como "medio de todos los medios", no puede ser sino co-

\footnotetext{
${ }^{7}$ Por ejemplo, cuando habla del gen: "El doble aspecto del gen consiste en regir varios caracteres a la vez, y en actuar sólo en relación con otros genes. El conjunto constituye un virtual, un potencial; y esa estructura se encarna en los organismos actuales, tanto desde el punto de vista de su especificación, como de la diferenciación de sus partes, según ritmos que, precisamente, se llaman 'diferenciales'." (DELEUZE, 1993, p. 240); o cuando se refiere al huevo: "[...] los tipos de huevos se distinguen por orientaciones, ejes de desarrollo, velocidades y ritmos diferenciales, como primeros factores de la actualización de una estructura, que crean un espacio y un tiempo propios de lo que se actualiza." (DELEUZE, 1993, p. 277).
} 
originario respecto a los medios mismos: es el caos en tanto relación, el caos en su potencia de coordinarse con los medios. Entre ritmos y medios, entonces, se va componiendo el ritornelo, gran máquina abstracta que fabrica tiempo: "El tiempo como forma a priori no existe, el ritornelo es la forma a priori del tiempo, que cada vez fabrica tiempos diferentes." (DELEUZE; GUATTARI, 1980, p. 431).

En la conferencia de 1978 "Le temps musical", dedicada a Pierre Boulez, vemos cómo esta concepción fragmentaria del ritmo toma un sentido que nos interesa particularmente. Se habla del tiempo no pulsado o tiempo flotante, fundamental para la obra del músico francés, entendiéndolo como duración: "[...] un tiempo liberado de la medida, sea regular o irregular." (DELEUZE, 2015, p. 13). Duración que Deleuze, siendo consecuente con su lectura de Bergson, entiende de entrada como "[...] una multiplicidad de duraciones heterócronas, cualitativas, no coincidentes, no comunicantes." (DELEUZE, 2015, p. 15). El asunto es cómo articular estas duraciones entre sí, como ponerlas en relación. No puede hacerse por una medida, claramente, ni siquiera una asimétrica, que correspondería al tiempo pulsado. Por ello hay que aplicar una concepción molecular del ritmo, que como ya sabemos está mucho más cerca del Caos que del número:

Está el caso de los biólogos: cuando estudian los ritmos vitales de periodos de 24 horas renuncian a articularlos conforme a un patrón común, por muy complejo que sea, o a una secuencia de procesos, sino que invocan lo que ellos llaman una población de osciladores moleculares, de moléculas oscilantes acopladas, que aseguran la comunicación de ritmos o la transritmicidad. (DELEUZE, 2015, p. 15).

Ante la proliferación de duraciones, será necesario que haya también proliferación de ritmos, comunicación constante a una magnitud inferior a ellos que desarma continuamente sus puntos estables. Concluye Deleuze (2015, P. 27): "Es posible que los ritmos y las duraciones vitales no estén organizados ni medidos por una forma mental, sino que se articulen desde adentro, desde procesos moleculares que los atraviesan.”

Este vínculo íntimo entre las duraciones y los ritmos, entonces, debe entenderse siempre como actuando en una multiplicidad continua, en un nivel molecular donde duraciones y ritmos se dividen y reúnen sin llegar a volverse del todo contables o estructurados. Sobre esto se extiende Deleuze en sus cursos sobre cine, particularmente en una clase del 17 de noviembre 
de 1981. Allí señala que "[...] nada funcionaría en el sistema si la duración no tuviera ella misma un extraño poder de dividirse y de reunirse continuamente." (DELEUZE, 2009, p. 59). Esta división opera "[...] en tantos ritmos, en tantas sub-duraciones, como objetos en el espacio haya implicados por el movimiento", a la vez que se reúnen "[...] en una sola y misma duración" (DELEUZE, 2009, p. 59). La duración puede definirse, entonces, como un movimiento simultáneo de división y de reunión. Un poco más adelante se refuerza esta idea, haciendo explícita alusión al famoso pasaje del río y los pájaros en Durée et simultanéité:

¿Por qué hacen falta al menos tres flujos? Observen que esos tres flujos son una figura muy variable, pues a veces los capto en uno, especie de ensońación en que la continuidad de mi vida interior, el correr del agua y el pasar o el vuelo del pájaro tienden a unirse en un mismo ritmo; luego los hago tres. ¿Por qué siempre hacen falta tres? Porque estando dados dos, hace falta un tercero para encarnar la posibilidad de su simultaneidad o no, la posibilidad de que estén reunidos en un mismo tercero o que se dividan. Posibilidad de reunión o de división. (DELEUZE, 2009, p. 61).

Entendemos, entonces, que el ritmo no funciona al nivel de la repetición o la medida, sino más profundamente, en la diferenciación; que tiene una relación intrínseca con el caos, emergiendo a la vez directamente de él como medio de todos los medios y creándose singularmente a partir de estos, en cada cambio de código que realizan; que es capaz de articular las duraciones en el tiempo no pulsado, a un nivel molecular y múltiple; que, junto a la duración, sólo pueden entenderse cabalmente como multiplicidad que se reúne y se divide a sí misma, entre la consistencia y la inconsistencia. Sin embargo, sigue resultando confusa la relación entre duración y ritmo, por lo que diremos algo más al respecto.

\section{UNA COEXISTENCIA RÍTMICA PARA LAS DURACIONES}

Una primera distinción entre ambos conceptos consiste en el hecho de que para Deleuze la duración es un concepto propiamente bergsoniano; tiene la firma de Bergson, la cual Deleuze no intenta borrar ni modificar. En cambio, el concepto de ritmo es usado sólo de forma esporádica por Bergson, y Deleuze no lo asume como propiedad de él ni de nadie. Es un concepto no firmado. Esto permite una gran libertad en sus usos, como ya hemos visto. De este modo, cuando Deleuze habla más directamente sobre Bergson habría 
que entender que el ritmo es una especie de noción auxiliar a la duración, utilizada de forma más o menos libre e imprecisa. Pero cuando habla de ritmo, la duración aparece como un componente del concepto, que se identifica parcialmente con él y que resalta algunas de sus cualidades: en particular, su relación con la variación y la multiplicidad. La duración sería el componente bergsoniano del concepto de ritmo que propone Deleuze.

En segundo lugar, podemos entender que esta identificación parcial entre ritmo y duración se corresponde con el hecho de que ambos son modos de enunciar el movimiento. Ambos conceptos comparten ciertas características, como su relación con la multiplicidad, pero se distinguen en su orientación y especialmente en las problemáticas donde funcionan. Si el ritmo es un intermedio, intervalo comunicante y diferencial que describe una relación antes que una forma, la duración opera en primer lugar como una continuidad en diferenciación perpetua sobre sí misma. Si el ritmo se opone a la medida y surge del caos (que también de origen a los medios), la duración se opone al tiempo homogéneo y surge de una extrema tensión del movimiento (cuya extrema distensión de origen al espacio). Por tanto, no resulta descabellado recurrir a aspectos de un concepto para resolver algo en el otro concepto, en una operación transductiva.

Por último, hay una forma de relacionar los conceptos que implicaría una exterioridad entre duración y ritmo, estableciéndose entre ellos una coexistencia real, en el mismo plano de ser. Si la principal característica del ritmo es su ser-entre, y la de la duración es la continuidad del flujo, es fácil pensar que el ritmo puede ser lo que está entre las duraciones, permitiendo así sus diferenciaciones e interrelaciones. Así podemos entender, por ejemplo, el sorprendente pasaje de Matière et mémoire en el que se habla de distintos ritmos de duración, algunos a nuestro nivel, otros más rápidos (como la vibración de los colores) y otros más lentos (como los planetas o las montañas). Varias duraciones se agruparían en una franja rítmica precisa, lo que permitiría su cohabitación a un mismo nivel de realidad (BERGSON, 1990b, p. 279). Ahora bien, a pesar del paralelismo existente entre los esquemas, esto no significa que debamos identificar las duraciones con los medios. Hay que recordar que los medios se definen por una vibración o repetición periódica que garantiza su persistencia; es justamente lo opuesto a la duración bergsoniana, que dura en función de su variación continua. Claramente medio y duración pueden coincidir en lo real, pero es preciso discernir sus distintas orientaciones. 
Hemos dicho que en este tercer caso tiene lugar una coexistencia real (ya no sólo temática o conceptual) entre ritmo y duración. Corresponde ahora que nos preguntemos por los modos de esta relación, para lo cual recurriremos a la coexistencia en tanto variación del concepto deleuziano de ritmo. A pesar de que ya podemos afirmar que el ritmo se establece siempre en multiplicidad desde Différence et répétition (lo que se reafirma en Mille Plateaux), es en Francis Bacon, Logique de la sensation, de 1981, donde la coexistencia rítmica se desarrolla con la mayor precisión. Podríamos decir que en este texto el ritmo tiene una triple aparición: como el doble movimiento de sístole y diástole de la Figura pictórica, como coexistencia de las sensaciones más allá de la división de los sentidos, y como despliegue dinámico en forma activa, pasiva y testigo. Para efectos de nuestro argumento, sólo consideraremos las dos primeras, y especialmente el tránsito que va de la una a la otra.

Diástole y sístole son los movimientos de dilatación y contracción del corazón, y Deleuze los toma de Maldiney para analizar la relación de la Figura con la estructura que la rodea. Dice Deleuze (2003, p. 37-38):

Todo se reparte en diástole y sístole repercutidas en cada nivel. La sístole que encoge el cuerpo, y va de la estructura a la Figura; la diástole que lo esparce y lo disipa, de la Figura a la estructura. Pero ya hay una diástole en el primer movimiento, cuando el cuerpo se alarga para encerrarse mejor; y hay una sístole en el segundo movimiento cuando el cuerpo se contrae para escaparse; e incluso cuando el cuerpo se disipa, todavía queda contraído por las fuerzas que lo cogen de un bocado para devolverlo a su alrededor. La coexistencia de todos los movimientos en el cuadro: eso es el ritmo.

Este doble movimiento nos recuerda sin duda al de la duración bergsoniana, que en su zona de mayor tensión es espíritu, y en su zona de mayor distensión es materia. Pero hay otro doble movimiento propio de la duración que también se liga con éste: ese "extraño poder" para reunirse y dividirse, atravesando los objetos y disgregándose en cada uno de ellos, que Deleuze mencionaba en sus clases. Tal vez no es tan casual que se haya ocupado la palabra "ritmo" en aquel pasaje, ya que con él sucede lo mismo: muchos ritmos se congregan en uno a la vez que éste se reparte en muchos. Ser a la vez, o coexistencia de movimientos contrarios y magnitudes dispares: el ritmo consiste precisamente en esta doble dinámica, que podemos ver en la duración pero también en el propio ritmo, de modo recursivo. La Figura se vuelve rítmica cuando accede a este doble movimiento de sístole y 
diástole, así como la duración se vuelve rítmica cuando la consideramos en su constante reunión y división, esto es, en su multiplicidad.

Podríamos decir entonces que el ritmo es la coexistencia de la diástole y la sístole, movimientos que componen un cuadro. Pero hay otra imbricación entre estas nociones que es mucho más profunda. Se trata del poder que tiene la pintura para mostrar la unidad de los sentidos, llegando a una "Figura multisensible". El ritmo es en este contexto "[...] una potencia vital que desborda todos los dominios y los atraviesa” (DELEUZE, 2003, p. 46): la proto-sensación a un nivel más profundo que el de la separación de los sentidos, caldo de cultivo de cualquier sensación posible. El ritmo como sinestesia, que "[...] aparece como música cuando inviste el nivel auditivo, como pintura cuando inviste el nivel visual", y que "recorre un cuadro como recorre una música." (DELEUZE, 2003, p. 46). Lo interesante es que el fundamento de esta comprensión del ritmo como coexistencia de las sensaciones es el mismo esquema sístole-diástole: "[...] el mundo que me atrapa cerrándose sobre mí, el yo que se abre al mundo, y que el propio yo abre." (DELEUZE, 2003, p. 46). El fundamento rítmico de la sensación está en este traspaso de un esquema propio de la figura pictórica al propio cuerpo sensorial, que deja de comprenderse como organismo para entenderse como latido de una potencia en el límite de lo vivible. Deleuze rompe en este punto con la fenomenología, pues considera que el cuerpo vivido es un punto de partida insuficiente: "La unidad del ritmo, en efecto, sólo podemos buscarla aquí donde el propio ritmo [se] sumerge en el caos, en la noche, y donde las diferencias de nivel están perpetuamente batidas con violencia." (DELEUZE, 2003, p. 47). Por eso requiere invocar una vez más la figura del cuerpo sin órganos, campo de puras intensidades, umbrales y niveles: si hay latido este no será del corazón como órgano, sino del cuerpo como potencia.

Nos queda revisar las consecuencias que este doble cruce entre coexistencia y lo que hemos llamado latido pueden tener para la relación entre ritmo y duración. En base al primer cruce, podemos imaginar que la duración ocupa el lugar de la Figura: el ritmo vendría, entonces, a modular la relación de una duración con su fondo mediante el doble movimiento de sístole y diástole (o, volviendo a Bergson, de tensión y distensión). La duración se contrae, definiéndose como unidad de variación continua, a la vez que se dilata, abriéndose a la mezcla, el acople o la participación con otras duraciones. Entra, así, en una segunda dinámica: la de reuniones y divisiones, que se hace posible por la presencia del ritmo como campo, 
simultáneamente, relacionador y diferenciador, un constante entremedio que rehúye de la determinación. En el primer caso, un ritmo como punto de partida singular, dinámica interna de cada duración; en el segundo, el ritmo como potencia relacionadora en general, dinámica externa que permite las constantes reagrupaciones de las duraciones entre sí.

La simultaneidad de flujos, entonces, desarrollada por Bergson en Durée et simultanéité en función de un Tiempo único e impersonal, puede entenderse también en función del ritmo: basta con que lo aprehendamos como relación de duraciones, la que puede entenderse en su sentido mínimo (relación de una duración con su entorno, o incluso consigo misma) o en su sentido máximo (relación de todas las duraciones entre sí). El ritmo como la coexistencia de todas las duraciones, pero fuera de todo cuadro también. Si esta comprensión es compatible con la hipótesis de un Tiempo único, o más bien la desbarata o reformula, es una discusión que tendremos que dejar para otro momento; por ahora sólo podemos esbozar una conjetura al respecto.

Se trata de una apreciación sobre el desajuste que hay entre las nociones de simultaneidad y coexistencia. La simultaneidad, aunque sea de flujos o movimientos, tiene como base la simultaneidad de instantes, la eternidad de un corte total y liso del universo: si pensamos este corte como corte móvil (lo que hace Deleuze en L'image-mouvement), seguimos conservando el esquema de una comunicación instantánea entre una duración y otra, en lugar de una comunicación duracional en sí misma. Por eso, lo que proponemos tal vez se explica mejor por la noción de coexistencia, que permite dar cuenta del carácter no-instantáneo de las relaciones sin por eso replegarse en situaciones atomizadas. Si es posible pensar en un Todo (lo que no está de ninguna manera decidido), este tendría que considerar los diferimientos insalvables entre las infinitas duraciones que lo componen: ni pura sucesión ni pura simultaneidad, sino más bien un ser en desfase, antes de la división entre lo temporal y lo espacial. Una coexistencia, en fin, que no tendría más base ontológica que la multiplicidad misma donde aquella se implica.

DURÁN, Cristóbal; KONG, Felipe. A rhythmic coexistence of duration: between Bergson and Deleuze. Trans/form/ação, Marília, v. 40, n. 2, p. 175-192, Abr./Jun., 2017. 
coexistence of heterogeneous levels of multiplicity. Although this concept has been thought of from a point of view that could be characterized as "continuist", Bergson himself avoids confusing this continuity with the idea of homogeneity. We will try to show that the determination of the concept of durée is reached by defining it as a tension or interlacement between succession and simultaneity, and therefore as a multiplicity based on the coexistence of different flows of duration. Bergson proposes the existence of an impersonal and unique time as the basis for the relationship between durations. Our proposal is that this same situation can be better understood through the concept of rhythm as articulated in Deleuze's work, a concept closely linked to the notions of Durée and coexistence. Through the movements of contraction, dilation, reunion, and division, rhythm operates along with the available durations as a field of relation, being able to occupy the place that had been assigned to unique time.

KeYwords: Durée. Bergson. Deleuze. Coexistence. Rhythm. Simultaneity.

\section{REFERÊNCIAS}

BACHELARD, G. La dialectique de la durée. Paris: Presses Universitaires de France, 2013.

BÉGUIN, A.; THÉVENAZ, P. Henri Bergson: essais et témoignages inédits. Neuchâtel: Éditions de la Baconnière, 1943.

BERGSON, H. Durée et simultanéité: à propos de la théorie d'Einstein. 7.éd. Paris: Presses Universitaires de France, 1968.

- La pensée et le mouvant: essais et conférences. Paris: Presses Universitaires de France, 1990a.

- Matière et mémoire: essai sur la relation du corps à l'esprit. Paris: Presses Universitaires de France, 1990b.

- Essai sur les données immédiates de la conscience. Paris: Presses Universitaires de France, 1991.

CORBIER, C. Bachelard, Bergson, Emmanuel. Mélodie, rythme et durée. Archives de Philosophie, n. 75, p. 291-310, 2012.

DELEUZE, G. Le Bergsonisme. Paris: Presses Universitaires de France, 1966.

. Cinéma 1. L’image-mouvement. Paris: Éditions de Minuit, 1983.

. Différence et répétition. Paris: Presses Universitaires de France, 1993.

. Proust et les signes. Paris: Presses Universitaires de France, 1998.

. Francis Bacon: logique de la sensation. Paris: Éditions du Seuil, 2003.

. Cine I: Bergson y las imágenes. Buenos Aires: Cactus, 2009.

. El tiempo musical. Ed. bilingue. Ciudad de México: El latido de la máquina, 2015. 
.; GUATTARI, F. Mille plateaux : capitalisme et schizophrénie. Paris: Éditions de Minuit, 1980.

DURING, É. L'espace-temps entre algèbre et géométrie: la théorie de la relativité chez Bergson et Bachelard. In: WORMS, F.; WUNENBURGER, J.-J. (Dir.), Bachelard et Bergson: continuité et discontinuité. Paris: Presses Universitaires de France, 2008. p. 135149.

. Introduction au dossier critique. In: BERGSON, H. Durée et simultanéité. Paris: Presses Universitaires de France, 2009. p. 219-244.

PARR, A. (Ed.). The Deleuze Dictionary. Edinburgh: Edinburg University Press, 2005.

SASSO, R.; VILLANI, A. (Ed.). Le vocabulaire de Gilles Deleuze. Paris: Les Cahiers de Noesis, 2003

YOUNG, E. et al. The Deleuze and Guattari Dictionary. New York: Bloomsbury, 2013.

ZOURABICHVILI, F. Le vocabulaire de Deleuze. Paris: Ellipses, 2003.

Recebido em 20/02/2017

Aceito em 28/02/2017 